\title{
Research Article \\ Existence of a Nonautonomous SIR Epidemic Model with Age Structure
}

\author{
Junyuan Yang ${ }^{1,2}$ and Xiaoyan Wang ${ }^{2}$ \\ ${ }^{1}$ Department of Applied Mathematics, Yuncheng University, Yuncheng Shanxi 044000, China \\ ${ }^{2}$ Beijing Institute of Information and Control, Beijing 100037, China
}

Correspondence should be addressed to Junyuan Yang, yangjunyuan00@126.com

Received 15 December 2009; Accepted 1 February 2010

Academic Editor: Toka Diagana

Copyright (c) 2010 J. Yang and X. Wang. This is an open access article distributed under the Creative Commons Attribution License, which permits unrestricted use, distribution, and reproduction in any medium, provided the original work is properly cited.

A nonautonomous SIR epidemic model with age structure is studied. Using integro-differential equation and a fixed point theorem, we prove the existence and uniqueness of a positive solution to this model. We conclude our results and discuss some problems to this model in the future. We simulate our analyzed results.

\section{Introduction}

Age structure of a population affects the dynamics of disease transmission. Traditional transmission dynamics of certain diseases cannot be correctly described by the traditional epidemic models with no age-dependence. A simplemodel was first proposed by Lotka and Von Foerster [1, 2], where the birth and the death processes were independent of the total population size and so the limitation of the resources was not taken into account. To overcome this deficiency, Gurtin and MacCamy [3], in their pioneering work considered a nonlinear age-dependent model, where birth and death rates were function of the total population. Various age-structured epidemic models have been investigated by many authors, and a number of papers have been published on finding the threshold conditions for the disease to become endemic, describing the stability of steady-state solutions, and analyzing the global behavior of these age-structured epidemic models (see [4-7]). We may find that the epidemic models that most authors discussed mainly include S-I-R that is, the total population of a country or a district was subdivided into two or three compartments containing susceptibles, infectives, or immunes; it was assumed that there is no latent class, so a person who catches 
the disease becomes infectious instantaneously. The basic SIR age-structured epidemic model is like the following equations:

$$
\begin{gathered}
\frac{\partial s(a, t)}{\partial t}+\frac{\partial s(a, t)}{\partial a}=-\lambda(a, t, i) s(a, t)-\mu(a) s(a, t), \\
\frac{\partial i(a, t)}{\partial t}+\frac{\partial i(a, t)}{\partial a}=\lambda(a, t, i) s(a, t)-\mu(a) i(a, t)-\gamma(a) i(a, t), \\
\frac{\partial r(a, t)}{\partial t}+\frac{\partial r(a, t)}{\partial a}=\gamma(a) i(a, t)-\mu(a) r(a, t), \\
s(0, t)=\int_{0}^{\infty} \beta(a) p(a, t) d a, \quad i(0, t)=r(0, t)=0, \\
s(a, 0)=s_{0}(a) \in L_{+}^{1}(0,+\infty), \quad i(a, 0)=i_{0}(a) \in L_{+}^{1}(0,+\infty), \\
r(0, a)=r_{0}(a) \in L_{+}^{1}(0,+\infty) .
\end{gathered}
$$

The non-autonomous phenomenon is so prevalent and all pervasive in the real life that modelling biological proceeding under non-autonomous environment should be more realistic than autonomous situation. The non-autonomous phenomenon is so prevalent in the real life that many epidemiological problems can be modeled by non-autonomous systems of nonlinear differential equations [8-11], which should be more realistic than autonomous differential equations. In one case, the incidence of many infectious diseases fluctuates over time and often exhibits periodic behavior. The basic SIR model is formulated by

$$
\begin{gathered}
\frac{d S}{d t}=B(t)-\mu(t) S-\alpha(t) S I, \\
\frac{d I}{d t}=\alpha(t) S I-\mu(t) I-\gamma(t) I, \\
\frac{d R}{d t}=\gamma(t) I-\mu(t) R .
\end{gathered}
$$

These works were mainly concerned with finding threshold conditions for the disease to become endemic and describing the stability of steady-state solutions, often under the assumption that the population has reached its steady state and the diseases do not affect the death rate of the population.

However, all of the models which are not mixed age structure and non-autonomous are only concluding age structure or non-autonomous. Birth rate or input function is dependent on age or dependent on time $t$ in these models cited therein. In fact, birth rate or input function is dependent not only on age $a$ and time $t$ but also on the total population $P(t)$. We know the resource is limited. As recognized by authors, there was only one paper $[3,12]$ related them. In $[3,12]$, their model are two dimensions about epidemic dynamics. The population is increasing year after year. The birth rate is a decrease function until the population attend certain level such as Logistic growth rate. At the same time, the death rate should be dependent on the total population $P(t)$. We can consider now more realistic and complex models in which the epidemic acts in a different way on infected, susceptible and recovered (immune). We consider a well-known expression for the force of infection which is 
justified in the literature. We choose as $L^{1}\left(R_{+}\right)$the natural space for the solution because the total population is finite.

This paper is organized as follows: Section 2 introduces a non-autonomous SIR model with age structure. In Section 3, existence and uniqueness of a solution for an epidemic model with different mortality rates on any finite time-interval is obtained. In Section 4 , we conclude our results and discuss the defect of our model.

\section{The Model Formulation}

This section describes the basic model we are going to analyze in this paper. The population is divided into three subclasses: susceptible, infected, and recovered. Where $S(a, t), I(a, t), R(a, t)$ denote the associated density functions with these respective epidemiological age-structured classes. Let $\mu_{i}(a, t, P(t)), i=1,2,3$, be the age-specific mortality of the susceptible, the infective and the recovered individuals at time $t$, respectively. We assume that the disease affects the death rate, so we have $\mu_{2}(a, t, P(t)) \geq \mu_{1}(a, t, P(t))$, and $\mu_{2}(a, t, P(t)) \geq \mu_{3}(a, t, P(t))$. We assume that all new born are susceptible whose birth process is described by

$$
s(0, t)=\int_{0}^{+\infty} \beta(a, t, P(t)) s(a, t) d a,
$$

where $\beta$ is the birth rate. We also suppose that the initial age distributions are given by $s_{0}, i_{0}$, and $r_{0}$. And the age-specific recovery rate, $\gamma$, is independent of the time. Then the joint dynamics of the age-structured epidemiological model for the transmission of SIR can be written as

$$
\begin{gathered}
\frac{\partial s(a, t)}{\partial t}+\frac{\partial s(a, t)}{\partial a}=-\lambda(a, t, i) s(a, t)-\mu_{1}(a, t, P(t)) s(a, t), \\
\frac{\partial i(a, t)}{\partial t}+\frac{\partial i(a, t)}{\partial a}=\lambda(a, t, i) s(a, t)-\mu_{2}(a, t, P(t)) i(a, t)-\gamma(a) i(a, t), \\
\frac{\partial r(a, t)}{\partial t}+\frac{\partial r(a, t)}{\partial a}=\gamma(a) i(a, t)-\mu_{3}(a, t, P(t)) r(a, t), \\
s(0, t)=\int_{0}^{\infty} \beta(a, t, P(t)) p(a, t) d a, \quad i(0, t)=r(0, t)=0, \\
s(a, 0)=s_{0}(a) \in L_{+}^{1}(0,+\infty), \quad i(a, 0)=i_{0}(a) \in L_{+}^{1}(0,+\infty), \\
r(0, a)=r_{0}(a) \in L_{+}^{1}(0,+\infty) .
\end{gathered}
$$

We supposes $s(a, t), i(a, t)$, and $r(a, t)$ belong to $W^{1,1}(0,+\infty)$. So, $s(a, t), i(a, t)$, and $r(a, t) \rightarrow$ 0 , as $a \rightarrow+\infty$. It is logical to satisfy the biological meaning. The horizontal transmission of the disease occurs according to the following law:

$$
\lambda(a, t, i)=\int_{0}^{+\infty} K\left(a, a^{\prime}\right) i\left(t, a^{\prime}\right) d a^{\prime}
$$


where $K\left(a, a^{\prime}\right)$ is the rate at which an infective individual of age $a^{\prime}$ comes into a disease transmitting contact with a susceptible individual of age $a$. Summing the equations of (2.2), we obtain the following problem for the population density $P(a, t)=S(a, t)+E(a, t)+I(a, t)$.

$$
P(t)=S(t)+I(t)+R(t)=\int_{0}^{\infty} s(a, t) d a+\int_{0}^{\infty} i(a, t) d a+\int_{0}^{\infty} r(a, t) d a .
$$

In this paper, we prove the existence and uniqueness of a nonnegative solution of the model (2.2) on any finite time-interval. Our results are based on a process of the agedependent problem for the susceptible the infected and the removed, and then a fixed point method. To study existence and uniqueness of a solution for an epidemic model with different mortality rates, we need the following hypotheses. Given $T>0$, we denote $I:=[0, T]$ and we suppose that

$\left(H_{1}\right)$ for $i=1,2,3, \mu_{i}(a, t, P)$ is a nonnegative measurable function such that the mapping $l \mid \rightarrow \mu_{i}(l, l+u, P)$ belongs to $L_{\mathrm{Loc}}^{1}\left(R_{+}\right)$for almost all $(u, P) \in R_{2}$. Moreover, there exists a constant $C_{1}(T)>0$ such that for all $P, P^{\prime} \in R$,

$$
\left|\mu_{i}(a, t, P)-\mu_{i}\left(a, t, P^{\prime}\right)\right| \leq C_{1}(T)\left|P-P^{\prime}\right|, \quad \text { a.e. }(a, t) \in R_{+} \times I .
$$

With the notation $\widehat{\mu}_{1}=\mu_{2}-\mu_{1}, \widehat{\mu}_{2}=\mu_{2}-\mu_{3}$, there exists another constant $C_{i}(T)>0$, $j=2,3$, such that

$$
\left|\widehat{\mu}_{i}(a, t, P)\right| \leq C_{j}(T) \log (|P|+e), \quad \text { a.e. }(a, t) \in R_{+} \times I, i=2,3 \text {. }
$$

$\left(H_{2}\right) \beta(a, t, P)$ is a nonnegative measurable function which has compact support on the variable $a$ and such that for all $P, P^{\prime} \in R$,

$$
\left|\beta(a, t, P)-\beta\left(a, t, P^{\prime}\right)\right| \leq C_{4}(T)\left|P-P^{\prime}\right|, \quad \text { a.e. }(a, t) \in R_{+} \times I,
$$

where $C_{4}(T)>0$ is another constant which depends only on $T$. Moreover, there exists a constant $C_{5}(T)>0$ such that for all $P \in R$,

$$
|\beta(a, t, P)| \leq C_{5}(T) \log (|P|+e) \text {, a.e. }(a, t) \in R_{+} \times I .
$$

$\left(H_{3}\right) \phi_{0}:=\left(s_{0}, i_{0}, r_{0}\right) \in\left(L^{1}\left(R_{+}\right)\right)^{3}$ has a compact support.

$\left(H_{4}\right) \gamma(a) \in L^{\infty}\left(R_{+}\right)$has compact support and is a nonnegative function. We set $\gamma_{\infty}=$ $\operatorname{ess} \sup _{a \in(0, \infty)} \gamma(a)$.

$\left(H_{5}\right) K\left(a, a^{\prime}\right) \in L^{\infty}\left(R_{+} \times R_{+}\right)$has a compact support and is a nonnegative function. We have $K_{\infty}=\operatorname{ess} \sup _{a \in(0, \infty)} K\left(a, a^{\prime}\right)$.

To simplify the calculation of estimates, we perform the change

$$
\begin{gathered}
i(a, t)=p(a, t)-s(a, t)-r(a, t), \\
\widehat{\mu}_{1}=\mu_{2}-\mu_{1}, \quad \widehat{\mu}_{2}=\mu_{2}-\mu_{3} .
\end{gathered}
$$


We obtain that the following system is analogous to (2.2).

$$
\begin{gathered}
\frac{\partial p(a, t)}{\partial t}+\frac{\partial p(a, t)}{\partial a}=-\widehat{\mu}_{1} s(a, t)+\widehat{\mu}_{2} r(a, t)-\mu_{2}(a, t, P(t)) p(a, t), \\
\frac{\partial s(a, t)}{\partial t}+\frac{\partial s(a, t)}{\partial a}=-\lambda(a, t, p-s-r) s(a, t)-\mu_{1}(a, t, P(t)) i(a, t), \\
\frac{\partial r(a, t)}{\partial t}+\frac{\partial r(a, t)}{\partial a}=r(a)[p(a, t)-r(a, t)]-\left(\mu_{3}(a, t, P(t))+\gamma(a)\right) r(a, t), \\
p(0, t)=s(0, t)=\int_{0}^{\infty} \beta(a, t, P(t)) p(a, t), \quad r(0, t)=0, \\
p(a, 0)=s_{0}(a) \in L_{+}^{1}(0,+\infty), \quad s(a, 0)=i_{0}(a) \in L_{+}^{1}(0,+\infty), \\
r(0, a)=r_{0}(a) \in L_{+}^{1}(0,+\infty),
\end{gathered}
$$

where

$$
\rho_{0}(a)=\left(p_{0}(a), s_{0}(a), r_{0}(a)\right)=\left(s_{0}(a)+r_{0}(a)+i_{0}(a), s_{0}(a), r_{0}(a)\right), \quad \text { a.e, } a \in(0,+\infty) \text {. }
$$

For biological reasons, we are interested in nonnegative solutions, so we consider that

$$
p(a, t) \geq s(a, t), \quad p(a, t) \geq r(a, t) .
$$

And we will look for solutions to (2.10) belonging to the following space:

$$
\begin{gathered}
V:=\left\{\rho \in L^{\infty}\left(I,\left(L^{1}\left(R_{+}\right)^{3}\right)\right) \mid \rho_{1}(a, t) \geq \rho_{2}(a, t) \geq 0,\right. \\
\left.\rho_{1}(a, t) \geq \rho_{2}(a, t) \geq 0, \text { a.e, }(a, t) \in R_{+} \times I\right\}
\end{gathered}
$$

endowed with the norm

$$
|\rho|_{1}=\operatorname{ess} \sup _{t \in I} e^{-k t}|\rho(\cdot, t)|_{1}
$$

where $k$ is a positive constant which will be chosen later and $|\cdot|_{1}$ denotes the usual norm in $L^{1}\left(R_{+}\right)$that is, $|\rho(\cdot, t)|_{1}=\left\|\rho_{1}(\cdot, t)\right\|_{L^{1}}+\left\|\rho_{2}(\cdot, t)\right\|_{L^{1}}+\left\|\rho_{3}(\cdot, t)\right\|_{L^{1}}$.

Namely, by a solution to $(2.10)$, we mean a function

$$
\rho(\cdot, \cdot)=(p(\cdot, \cdot), s(\cdot, \cdot), r(\cdot, \cdot)) \in V,
$$


such that

$$
\begin{gathered}
D p=-\widehat{\mu}_{1} s(a, t)+\widehat{\mu}_{2} r(a, t)-\mu_{2}(a, t, P(t)) p(a, t), \\
D s=-\lambda(a, t, p-s-r) s(a, t)-\mu_{1}(a, t, P(t)) i(a, t), \\
D r=\gamma(a)[p(a, t)-r(a, t)]-\left(\mu_{3}(a, t, P(t))+\gamma(a)\right) r(a, t), \\
\lim _{t \rightarrow 0^{+}} p(a, t+h)=\int_{0}^{\infty} \beta(a, t, P(t)) p(a, t) d a, \\
\lim _{t \rightarrow 0^{+}} s(a, t+h)=\int_{0}^{\infty} \beta(a, t, P(t)) p(a, t) d a, \\
\lim _{t \rightarrow 0^{+}} r(a, t+h)=0, \\
p(a, t), s(a, t), r(a, t) \longrightarrow 0, \quad \text { when } a \longrightarrow+\infty .
\end{gathered}
$$

In order to prove the existence of solution of (2.10), adding $\gamma(a)$ in both sides of (2.16) in technical style, we have

$$
D p=-\widehat{\mu}_{1} s(a, t)+\widehat{\mu}_{2} r(a, t)+\gamma(a) p(a, t)-\left(\mu_{2}(a, t, P(t))+\gamma(a)\right) p(a, t),
$$

where $D p, D s$, and $D r$ denote the directional derivatives of $p, s$ and $r$, respectively, that is,

$$
D p(a, t)=\lim _{h \rightarrow 0} \frac{p(a+h, t+h)-p(a, t)}{h} .
$$

Generally, $\rho$ will not be differentiable everywhere; of course,when this occurs, $D p=\partial p / \partial a+$ $\partial p / \partial t, D s=\partial s / \partial a+\partial s / \partial t$ and $D r=\partial r / \partial a+\partial r / \partial t$.

\section{Existence of a Solution to the System}

If we assume that $\rho=(p, s, r)$ is smooth along the characteristics $a=t+c$ (except perhaps for a zero-measure set of $c$ ), considering

$$
B_{p}=\int_{0}^{\infty} \beta(a, t, P(t)) p(a, t) d a,
$$


where $P(t)=\int_{0}^{+\infty} p(a, t) d a$, and integrating equalities of (2.16) along the line, we obtain the following ODS

$$
p(a, t)=\left\{\begin{array}{l}
p_{0}(a-t) \pi_{1}(a, t, t, \rho) \\
\quad+\int_{0}^{t} \pi_{1}(a, t, \sigma, \rho)\left[\widehat{\mu}_{1}(a-\sigma, t-\sigma, P(t-\sigma)) s(a-\sigma, t-\sigma)\right. \\
\left.\quad+\widehat{\mu}_{2}(a-\sigma, t-\sigma, P(t-\sigma)) r(a-\sigma, t-\sigma)\right] d \sigma, \quad a \geq t, \\
B_{p}(t-a) \pi_{1}(a, t, a, \rho) \\
\quad+\int_{0}^{a} \pi_{1}(a, t, \sigma, \rho)\left[\widehat{\mu}_{1}(a-\sigma, t-\sigma, P(t-\sigma)) s(a-\sigma, t-\sigma)\right. \\
\left.\quad+\widehat{\mu}_{2}(a-\sigma, t-\sigma, P(t-\sigma)) r(a-\sigma, t-\sigma)\right] d \sigma, \quad a<t .
\end{array}\right.
$$

Integrating (2.7) along $a=t+c$, we also get $p(a, t)$ for technical need

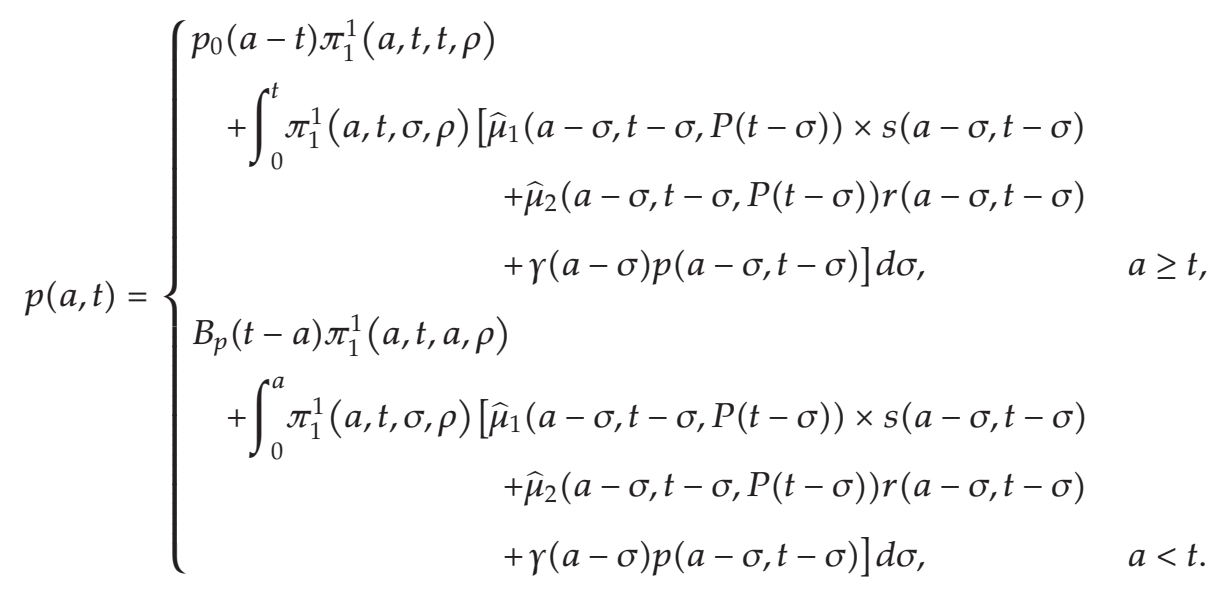

Integrating the second equation of (2.16) along $a=t+c$, we have

$$
s(a, t)= \begin{cases}s_{0}(a-t) \pi_{2}(a, t, t, \rho), & a \geq t, \\ B_{p}(t-a) \pi_{2}(a, t, a, \rho), & a<t .\end{cases}
$$

Integrating the third equation of (2.16) along $a=t+c$, we obtain

$$
r(a, t)=\left\{\begin{aligned}
r_{0}(a-t) \pi_{3}(a, t, t, \rho) & \\
& \quad+\int_{0}^{t} \pi_{3}(a, t, \sigma, \rho) \gamma(a-\sigma)(p(a-\sigma, t-\sigma)-s(a-\sigma, t-\sigma)) d \sigma, \quad a \geq t \\
& B_{p}(t-a) \pi_{1}^{1}(a, t, a, \rho) \\
& \quad+\int_{0}^{a} \pi_{3}(a, t, \sigma, \rho) \gamma(a-\sigma)(p(a-\sigma, t-\sigma)-s(a-\sigma, t-\sigma)) d \sigma, \quad a<t
\end{aligned}\right.
$$


where

$$
\begin{gathered}
\pi_{1}(a, t, x, \rho)=\exp \int_{0}^{x} \mu_{2}(a-s, t-s, P(t-s)) d s \\
\pi_{2}(a, t, x, \rho)=\exp \int_{0}^{x}\left(\mu_{1}(a-s, t-s, P(t-s))+\lambda(a-\sigma, t-\sigma, p-s-r)\right) d s \\
\pi_{3}(a, t, x, \rho)=\exp \int_{0}^{x}\left(\mu_{1}(a-s, t-s, P(t-s))+\gamma(a-\sigma)\right) d s \\
\pi_{1}^{1}(a, t, x, \rho)=\exp \int_{0}^{x}\left(\mu_{2}(a-s, t-s, P(t-s))+\gamma(a-\sigma)\right) d s
\end{gathered}
$$

We can easily see that solving (2.16) is equivalent to finding a solution to (3.2), (3.4) and (3.5) or (3.3), (3.4), and (3.5) (see [3]). So, in the sequel, we restrict our attention to these integral equations.

Let us consider $r=\log \left(\left|\rho_{0}\right|_{1}+e\right)$ with $\rho_{0}$, and $w>0$ fixed. Consider the set

$$
C_{r, w}=\left\{\left.\rho \in V|| \rho(\cdot, t)\right|_{1} \leq \exp \left(r e^{w t}\right) \text { a.e. } t \in I\right\} .
$$

The following result provides some useful estimates.

Lemma 3.1. Suppose $\left(H_{1}\right)-\left(H_{5}\right)$, and let $\rho:=(p, s, r), \rho^{\prime}:=\left(p^{\prime}, s^{\prime}, r^{\prime}\right) \in C_{r, w}, a \in R_{+}$, and $t \in I$. Then for $x \leq \min \{a, t\}$,

(i)

$$
\left|\pi_{i}(a, t, x ; \rho)\right| \leq 1, \quad i=1,2,3 .
$$

(ii) $\exists M(T)>0$ such that

$$
|P(t)|,\left|B_{p}(t)\right| \leq M(T) \quad \text { a.e. } t \in I \text {. }
$$

(iii) $\exists C_{j}(T)>0$, such that

$$
\left|\pi_{i}(a, t, x ; \rho)-\pi_{i}(a, t, x ; \rho)\right| \leq \frac{C_{j}(T)}{k}\left|\rho-\rho^{\prime}\right|_{V} e^{k t}, \quad i=1,3, j=6,7 .
$$

(iv) $\exists C\left(T, K_{\infty}\right)>0$, such that

$$
\left|\pi_{2}(a, t, x ; \rho)-\pi_{2}\left(a, t, x ; \rho^{\prime}\right)\right| \leq \frac{C\left(T, K_{\infty}\right)}{k}\left|\rho-\rho^{\prime}\right|_{V} e^{k t}
$$


Proof. Firstly, note that (3.8) and (3.9) are immediate. On the other hand,

$$
\begin{aligned}
& \left|\pi_{i}(a, t, x ; \rho)-\pi_{i}(a, t, x ; \rho)\right| \\
& \leq \int_{0}^{x}\left|\mu_{i}(a-s, t-s, P(t-s))-\mu_{i}\left(a-s, t-s, P^{\prime}(t-s)\right)\right| d s \\
& \leq C_{j}(T) \int_{0}^{x}\left|P(t-s)-P^{\prime}(t-s)\right| d s \\
& =C_{j}(T) \int_{t-x}^{t}\left|P(s)-P^{\prime}(s)\right| d s \\
& =C_{j}(T) \int_{t-x}^{t}\left|p(s, \cdot)-p^{\prime}(s, \cdot)\right|_{L^{1}} d s \\
& \leq \frac{C_{j}(T)}{k}\left|\rho-\rho^{\prime}\right|_{V} e^{k t}, \quad i=1,3, j=6,7, \\
& \left|\pi_{2}(a, t, x ; \rho)-\pi_{2}(a, t, x ; \rho)\right| \\
& \leq \int_{0}^{x}\left|\mu_{1}(a-s, t-s, P(t-s))-\mu_{1}\left(a-s, t-s, P^{\prime}(t-s)\right)\right| d s \\
& +\int_{0}^{x}\left|\lambda(a-s, t-s ; p-s-r)-\lambda\left(a-s, t-s, p^{\prime}-s^{\prime}-r^{\prime}\right)\right| d s \\
& \leq C_{1}(T) \int_{0}^{x}\left|P(t-s)-P^{\prime}(t-s)\right| d s+C\left(T, K_{\infty}\right) \int_{0}^{x}\left|\rho-\rho^{\prime}\right|_{L^{1}} d s \\
& =C_{1}(T) \int_{t-x}^{t} e^{k t} e^{-k t}\left|p(s, \cdot)-p^{\prime}(s, \cdot)\right|_{L^{1}} d s+C\left(T, K_{\infty}\right) \int_{0}^{x} e^{k t} e^{-k t}\left|\rho-\rho^{\prime}\right|_{L^{1}} d s \\
& \leq \frac{C_{1}(T)}{k}\left|\rho-\rho^{\prime}\right|_{V} e^{k t}+\frac{C\left(T, K_{\infty}\right)}{k}\left|\rho-\rho^{\prime}\right|_{V} e^{k t} .
\end{aligned}
$$

We set $C_{1 K}=C_{1}(T)+C\left(T, K_{\infty}\right)$, and then

$$
\left|\pi_{2}(a, t, x ; \rho)-\pi_{2}(a, t, x ; \rho)\right| \leq \frac{C_{1 K}}{k}\left|\rho-\rho^{\prime}\right|_{V} e^{k t} .
$$

Lemma 3.2. Suppose $\left(H_{1}\right)-\left(H_{5}\right)$, if $\rho=(p, s, r) \in V$ satisfies (3.2), (3.4), and (3.5), or (3.3), (3.4), and (3.5), then there exists a constant $w>0$, depending only on $T$ and $\gamma_{\infty}$, such that $\rho \in C_{r, w}$ with $C_{r, w}$ defined in (3.7). 
Proof. Suppose that $\rho=(p, s, r) \in V$ satisfies the above assumptions. Considering (3.2), (3.4) and (3.5), or (3.3), (3.4), and (3.5), thanks to (3.7) and an obvious change of variables in the integrals, we have for all $t \in I$,

$$
\begin{aligned}
& |\rho(t, \cdot)|_{1} \\
& =\left\|\rho_{1}(t, \cdot)\right\|+\left\|\rho_{2}(t, \cdot)\right\|+\left\|\rho_{3}(t, \cdot)\right\| \\
& =\int_{t}^{\infty} p_{0}(a-t) \pi_{1}(a, t, t ; \rho) d a \\
& +\int_{t}^{\infty} \int_{0}^{t} \pi_{1}(a, t, \sigma ; \rho)\left[\widehat{\mu}_{1}(a-\sigma, t-\sigma, P(t-\sigma)) s(a-\sigma, t-\sigma)\right. \\
& \left.+\widehat{\mu}_{2}(a-\sigma, t-\sigma, P(t-\sigma)) r(a-\sigma, t-\sigma)\right] d \sigma d a \\
& +\int_{0}^{t} B_{p}(t-a) \pi_{1}(a, t, a ; \rho) d a \\
& +\int_{0}^{t} \int_{0}^{a} \pi_{1}(a, t, \sigma ; \rho)\left[\widehat{\mu}_{1}(a-\sigma, t-\sigma, P(t-\sigma)) s(a-\sigma, t-\sigma)\right. \\
& \left.+\widehat{\mu}_{2}(a-\sigma, t-\sigma, P(t-\sigma)) r(a-\sigma, t-\sigma)\right] d \sigma d a \\
& +\int_{t}^{\infty} s_{0}(a-t) \pi_{2}(a, t, t ; \rho) d a+\int_{0}^{t} B_{p}(t-a) \pi_{2}(a, t, a ; \rho) d a+\int_{t}^{\infty} r_{0}(a-t) \pi_{3}(a, t, t ; \rho) d a \\
& +\int_{t}^{\infty} \int_{0}^{t} \gamma(a-\sigma) \pi_{3}(a, t, \sigma ; \rho) \times[p(a-\sigma, t-\sigma)-s(a-\sigma, t-\sigma)] d \sigma d a \\
& +\int_{0}^{t} \int_{0}^{a} \gamma(a-\sigma) \pi_{3}(a, t, \sigma ; \rho) \times[p(a-\sigma, t-\sigma)-s(a-\sigma, t-\sigma)] d \sigma d a \\
& =\int_{t}^{\infty} \rho_{0}(a-t) \pi_{1}(a, t, t ; \rho) d a \\
& +\int_{0}^{t} \int_{t-\sigma}^{\infty} \pi_{1}(a+t-\sigma, t, t-\sigma ; \rho)\left[\widehat{\mu}_{1}(a, \sigma, P(\sigma)) s(a, \sigma)+\widehat{\mu}_{2}(a, \sigma, P(\sigma)) r(a, \sigma)\right] d a d \sigma \\
& +\int_{0}^{t} B_{p}(t-a) \pi_{1}(a, t, a ; \rho) d a \\
& +\int_{0}^{t} \int_{0}^{t-\sigma} \pi_{1}(a+t-\sigma, t, t-\sigma ; \rho)\left[\widehat{\mu}_{1}(a, \sigma, P(\sigma)) s(a, \sigma)+\widehat{\mu}_{2}(a, \sigma, P(\sigma)) r(a, \sigma)\right] d a d \sigma \\
& +\int_{0}^{t} B_{p}(t-a) \pi_{2}(a, t, a ; \rho) d a \\
& +\int_{0}^{t} \int_{t-\sigma}^{\infty} \gamma(a) \pi_{3}(a+t-\sigma, t, t-\sigma ; \rho)[p(a, \sigma)-s(a, \sigma)] d a d \sigma \\
& +\int_{0}^{t} \int_{0}^{t-\sigma} \gamma(a) \pi_{3}(a+t-\sigma, t, t-\sigma ; \rho) \times[p(a, \sigma)-s(a, \sigma)] d a d \sigma
\end{aligned}
$$




$$
\begin{aligned}
& \leq \int_{t}^{\infty}\left|\rho_{0}(a-t)\right| d a+\int_{0}^{t} \int_{0}^{\infty}\left|\widehat{\mu}_{1}(a, \sigma, P(\sigma)) s(a, \sigma)\right| d a d \sigma \\
& \quad+\int_{0}^{t} \int_{0}^{\infty}\left|\widehat{\mu}_{2}(a, \sigma, P(\sigma)) r(a, \sigma)\right| d a d \sigma \\
& \quad+2 \int_{0}^{t}\left|B_{p}(t-a)\right| d a+\int_{0}^{t} \int_{0}^{\infty}|\gamma(a) p(a, \sigma)-s(a, \sigma)| d a d \sigma \\
& \leq\left|\rho_{0}(a-t)\right|_{1}+\bar{\mu}_{1} \int_{0}^{t}\|s(\cdot, \sigma)\|_{L^{1}} d \sigma+\bar{\mu}_{1} \int_{0}^{t}\|r(a, \sigma)\|_{L^{1}} d \sigma \\
& \quad+2 \int_{0}^{t} \log (|P|+e)\|p(\cdot, \sigma)\|_{L^{1}} d \sigma+\gamma_{\infty} \int_{0}^{t}\|p(\cdot, \sigma)\|_{L^{1}}+\gamma_{\infty} \int_{0}^{t}\|s(\cdot, \sigma)\|_{L^{1}} d \sigma \\
& \leq\left|\rho_{0}(a-t)\right|_{1}+\left(2+\gamma_{\infty}\right) \int_{0}^{t} \log (|P|+e)\|p(\cdot, \sigma)\|_{L^{1}} d \sigma+\left(\bar{\mu}_{1}+\gamma_{\infty}\right) \int_{0}^{t}\|s(\cdot, \sigma)\|_{L^{1}} d \sigma \\
& \quad+\bar{\mu}_{2} \int_{0}^{t}\|r(a, \sigma)\|_{L^{1}} d \sigma \\
& \leq\left|\rho_{0}(a-t)\right|_{1}+\left(2+2 \gamma_{\infty}+\bar{\mu}_{1}+\bar{\mu}_{2}\right) \int_{0}^{t} \log (|P|+e)\|\rho(\cdot, \sigma)\|_{1} d \sigma .
\end{aligned}
$$

We use the Gronwall's inequality, and then

$$
|\rho(t, \cdot)|_{1} \leq\left|\rho_{0}\right|_{1} \exp w \int_{0}^{t} \log (|P|+e) d u,
$$

where $w=2+2 \gamma_{\infty}+\bar{\mu}_{1}+\bar{\mu}_{2}$ and $\widehat{\mu}_{i}(a, t, P(t)) \leq \bar{\mu}_{i}, i=1,2$.

Let us consider the map $\rho=\left(\rho_{1}, \rho_{2}, \rho_{3}\right) \in V \rightarrow F(\rho)=\left(F_{1}(\rho), F_{2}(\rho), F_{3}(\rho)\right) \in V$, where $F(\rho)$ is defined by

$$
F_{1}(\rho)(a, t)=\left\{\begin{array}{rr}
p_{0}(a-t) \pi_{1}(a, t, t ; \rho) & \\
+\int_{0}^{t} \pi_{1}(a, t, \sigma ; \rho)\left[\widehat{\mu}_{1}(a-\sigma, t-\sigma) \times F_{2}(\rho)(a-\sigma, t-\sigma)\right. & \\
& \left.+\widehat{\mu}_{2} \times F_{3}(\rho)(a-\sigma, t-\sigma)\right] d \sigma, \quad t<a, \\
B_{p}(t-a) \pi_{1}(a, t, a ; \rho) & \\
+\int_{0}^{a} \pi_{1}(a, t, \sigma ; \rho)\left[\widehat{\mu}_{1}(a-\sigma, t-\sigma) \times F_{2}(\rho)(a-\sigma, t-\sigma)\right. & \\
& \left.+\widehat{\mu}_{2} \times F_{3}(\rho)(a-\sigma, t-\sigma)\right] d \sigma, \quad
\end{array}\right.
$$


and also $F_{1}(\rho)$ can be equal to

$$
\begin{aligned}
& F_{1}(\rho)(a, t)=\left\{\begin{array}{rr}
p_{0}(a-t) \pi_{1}^{1}(a, t, t ; \rho) & \\
+\int_{0}^{t} \pi_{1}^{1}(a, t, \sigma ; \rho)\left[\widehat{\mu}_{1}(a-\sigma, t-\sigma) \times F_{2}(\rho)(a-\sigma, t-\sigma)\right. & \\
& \left.+\widehat{\mu}_{2} \times F_{3}(\rho)(a-\sigma, t-\sigma)\right] d \sigma, \\
& \\
B_{p}(t-a) \pi_{1}^{1}(a, t, a ; \rho) & t<a, \\
+\int_{0}^{a} \pi_{1}^{1}(a, t, \sigma ; \rho)\left[\widehat{\mu}_{1}(a-\sigma, t-\sigma) \times F_{2}(\rho)(a-\sigma, t-\sigma)\right. & \\
& \left.+\widehat{\mu}_{2} \times F_{3}(\rho)(a-\sigma, t-\sigma)\right] d \sigma, \quad t \geq a,
\end{array}\right. \\
& F_{2}(\rho)(a, t)= \begin{cases}s_{0}(a-t) \pi_{2}(a, t, t ; \rho), & t<a, \\
B_{p}(t-a) \pi_{2}(a, t, a ; \rho), & t \geq a,\end{cases}
\end{aligned}
$$

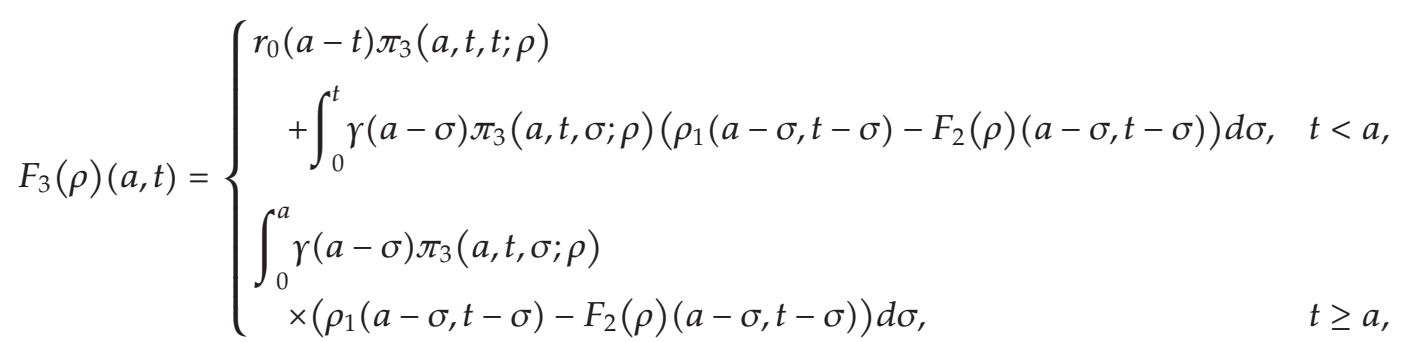

where $p(t)=\int_{0}^{\infty} \rho_{1}(a, t) d a$.

Lemma 3.3. With the assumptions of Lemma 3.2, we have $F: V \rightarrow V$.

Proof. In this proof we denote, for abbreviation,

$$
\begin{gathered}
\Gamma_{k}(x, y)=\exp \left(-\int_{x}^{y} \widehat{\mu}_{k}(a-s, t-s ; P(t-s)) d s\right), \quad k=1,2, \\
\Phi_{1}(x, y)=\exp \left(-\int_{x}^{y} \lambda(a-s, t-s ; p-s-r) d s\right), \\
\Phi_{2}(x, y)=\exp \left(-\int_{x}^{y} \gamma(a-s, t-s ; p-s-r) d s\right) .
\end{gathered}
$$

If $\rho \in V$, then $P(t) \in L^{\infty}(I)$. Then $\beta(a, t, P(t)), \widehat{\mu}_{i}(a, t, P(t)) \in L^{\infty}\left(R_{+} \times I\right), i=1,2$, by (2.5) and (2.7). Hence, $F$ is clearly measurable in $a$ and essentially bounded on $I$.

By (3.18), $F_{2}(\rho)(a, t) \geq 0$, a.e. $(a, t) \in R_{+} \times I$. So, we only need to show that $F_{1}(\rho)(a, t) \geq$ $F_{2}(\rho)(a, t), F_{1}(\rho)(a, t) \geq F_{3}(\rho)(a, t)$, a.e. $(a, t) \in R_{+} \times I, F_{3}(\rho) \geq 0$, and $F_{1}(\rho) \geq 0$, a.e. $(a, t) \in$ 
$R_{+} \times I$. We assume that $a \geq t$ (the discussion for $a<t$ is similar). Using (3.11) and (3.19) and substituting $F_{2}$ and $F_{3}$ into $F_{1}$ we get

$$
\begin{aligned}
& F_{1}(\rho)(a, t)-F_{2}(\rho)(a, t) \\
& =p_{0}(a-t) \pi_{1}(a, t, t, \rho)-s_{0}(a-t) \pi_{2}(a, t, t, \rho) \\
& \quad+\int_{0}^{t} \pi_{1}(a, t, \sigma, \rho)\left(\widehat{\mu}_{1}(a-\sigma, t-\sigma, P(t-\sigma)) F_{2}(\rho)(a-\sigma, t-\sigma)\right. \\
& \left.\quad+\widehat{\mu}_{2}(a-\sigma, t-\sigma, P(t-\sigma)) F_{3}(\rho)(a-\sigma, t-\sigma)\right) d \sigma \\
& =: p_{0}(a-t) \pi_{1}(a, t, t, \rho)-s_{0}(a-t) \pi_{2}(a, t, t, \rho)+A+B .
\end{aligned}
$$

Now, we proceed to estimate these quantities to see that $F_{1} \geq F_{2}$. By the mean value theorem, there exists $t_{1} \in(0, t)$, such that

$$
\begin{aligned}
A & =\int_{0}^{t} \pi_{1}(a, t, \sigma, \rho) \widehat{\mu}_{1}(a-\sigma, t-\sigma, P(t-\sigma)) F_{2}(\rho)(a-\sigma, t-\sigma) d \sigma \\
& =\int_{0}^{t} \pi_{1}(a, t, \sigma, \rho) \widehat{\mu}_{1}(a-\sigma, t-\sigma, P(t-\sigma)) s_{0}(a-t) \pi_{2}(a-\sigma, t-\sigma, t-\sigma, \rho) d \sigma \\
& =s_{0}(a-t) \pi_{2}(a, t, t, \rho)(1-\Gamma(0, t)) \Psi^{-1}\left(0, t_{1}\right)
\end{aligned}
$$

where

$$
\begin{aligned}
B= & \int_{0}^{t} \pi_{1}(a, t, \sigma, \rho) \widehat{\mu}_{2}(a-\sigma, t-\sigma, P(t-\sigma)) F_{3}(\rho)(a-\sigma, t-\sigma) d \sigma \\
= & \int_{0}^{t} \pi_{1}(a, t, \sigma, \rho) \widehat{\mu}_{2}(a-\sigma, t-\sigma, P(t-\sigma)) \\
& \times\left[r_{0}(a-t) \pi_{3}(a-\sigma, t-\sigma, t-\sigma, \rho) d \sigma\right. \\
& \left.\quad+\int_{\sigma}^{t} \gamma(a-s) \pi_{3}(a-\sigma, t-\sigma, s-\sigma, \rho)\left(\rho_{1}(a-s, t-s)-F_{2}(\rho)(a-s, t-s)\right)\right] d s d \sigma \\
= & r_{0}(a-t) \int_{0}^{t} \pi_{1}(a, t, \sigma, \rho) \widehat{\mu}_{2}(a-\sigma, t-\sigma, P(t-\sigma)) \pi_{3}(a-\sigma, t-\sigma, t-\sigma, \rho) d \sigma \\
& +\int_{0}^{t} \pi_{1}(a, t, \sigma, \rho) \widehat{\mu}_{2}(a-\sigma, t-\sigma, P(t-\sigma)) \\
& \times \int_{\sigma}^{t} \gamma(a-s) \pi_{3}(a-\sigma, t-\sigma, s-\sigma, \rho)\left(\rho_{1}(a-s, t-s)-F_{2}(\rho)(a-s, t-s)\right) d s d \sigma \\
=: & B_{1}+B_{2} .
\end{aligned}
$$


By the mean value theorem, there exists $t_{2} \in(0, t)$, such that

$$
\begin{aligned}
B_{1} & =r_{0}(a-t) \int_{0}^{t} \pi_{1}(a, t, \sigma, \rho) \widehat{\mu}_{2}(a-\sigma, t-\sigma, P(t-\sigma)) \pi_{3}(a-\sigma, t-\sigma, t-\sigma, \rho) d \sigma \\
& =r_{0}(a-t) \pi_{3}(a, t, t, \rho)\left(1-\Gamma_{2}(0, t)\right) \Psi_{2}^{-1}\left(0, t_{2}\right)
\end{aligned}
$$

and by the mean value theorem, there exists $t_{s} \in(0, t)$, such that

$$
\begin{aligned}
B_{2}= & \int_{0}^{t} \pi_{1}(a, t, \sigma, \rho) \widehat{\mu}_{2}(a-\sigma, t-\sigma, P(t-\sigma)) \\
& \times \int_{\sigma}^{t} \gamma(a-s) \pi_{3}(a-\sigma, t-\sigma, s-\sigma, \rho)\left(\rho_{1}(a-s, t-s)-F_{2}(\rho)(a-s, t-s)\right) d s d \sigma \\
= & \int_{0}^{t} \gamma(a-s)\left(\rho_{1}(a-s, t-s)-F_{2}(\rho)(a-s, t-s)\right) \int_{0}^{s} \pi_{1}(a, t, \sigma, \rho) \\
& \times \widehat{\mu}_{2}(a-\sigma, t-\sigma, P(t-\sigma)) \pi_{3}(a-\sigma, t-\sigma, s-\sigma, \rho) d \sigma d s \\
= & \int_{0}^{t} \gamma(a-s)\left(\rho_{1}(a-s, t-s)-F_{2}(\rho)(a-s, t-s)\right) \pi_{3}(a, t, s, \rho) \\
& \times \int_{0}^{s}-\frac{d}{d \sigma} \Gamma_{2}(0, \sigma) \Psi_{2}^{-1}(0, \sigma) d \sigma 2 d s \\
= & \int_{0}^{t} \gamma(a-s)\left(\rho_{1}(a-s, t-s)-F_{2}(\rho)(a-s, t-s)\right) \pi_{3}(a, t, s, \rho)\left(1-\Gamma_{2}(0, s)\right) \Psi_{2}^{-1}\left(0, t_{s}\right) .
\end{aligned}
$$

We substitute $A, B_{1}$, and $B_{2}$ into the formula of $F_{1}(\rho)(a, t)-F_{2}(\rho)(a, t)$. Thus,

$$
\begin{aligned}
F_{1}(\rho)(a, t)-F_{2}(\rho)(a, t) \\
=p_{0}(a-t) \pi_{1}(a, t, t, \rho)-s_{0}(a-t) \pi_{2}(a, t, t, \rho) \\
\quad+s_{0}(a-t) \pi_{2}(a, t, t, \rho)\left(1-\Gamma_{1}(0, t)\right) \Psi^{-1}\left(0, t_{1}\right) \\
\quad+r_{0}(a-t) \pi_{3}(a, t, t, \rho)\left(1-\Gamma_{2}(0, t)\right) \Psi_{2}^{-1}\left(0, t_{2}\right) \\
\quad+\int_{0}^{t} r(a-s)\left(\rho_{1}(a-s, t-s)-F_{2}(\rho)(a-s, t-s)\right) \\
\quad \times \pi_{3}(a, t, s, \rho)\left(1-\Gamma_{2}(0, s)\right) \Psi_{2}^{-1}\left(0, t_{s}\right) d s
\end{aligned}
$$




$$
\begin{aligned}
\geq & p_{0}(a-t) \pi_{1}(a, t, t, \rho)-s_{0}(a-t) \pi_{2}(a, t, t, \rho) \Gamma_{1}(0, t) \Psi^{-1}\left(0, t_{1}\right)+B_{1}+B_{2} \\
\geq & p_{0}(a-t) \pi_{1}(a, t, t, \rho)-s_{0}(a-t) \pi_{1}(a, t, t, \rho) \Psi_{1}\left(t_{1}, t\right)+B_{1}+B_{2} \\
\geq & p_{0}(a-t) \pi_{1}(a, t, t, \rho)\left(1-\Psi_{1}\left(t_{1}, t\right)\right)+B_{1} \\
& \quad+\int_{0}^{t} \gamma(a-s)\left(\rho_{1}(a-s, t-s)-F_{2}(\rho)(a-s, t-s)\right) \\
& \times \pi_{3}(a, t, s, \rho)\left(1-\Gamma_{2}(0, s)\right) \Psi_{2}^{-1}\left(0, t_{s}\right) d s \\
\geq & \left(p_{0}(a-t) \pi_{1}(a, t, t, \rho)\left(1-\Psi_{1}\left(t_{1}, t\right)\right)+B_{1}\right) e^{\int_{0}^{t} \gamma(a-s) \pi_{3}(a, t, s, \rho)\left(1-\Gamma_{2}(0, s)\right) \Psi_{2}^{-1}\left(0, t_{s}\right)} \\
\geq & 0 .
\end{aligned}
$$

By the formula of $F_{3}(\rho)$, we have $F_{3}(\rho) \geq 0$. Using (3.17) and (3.18) and substituting $F_{2}$ and $F_{3}$ into $F_{1}$ we get

$$
\begin{aligned}
F_{1}(\rho)(a, t)-F_{3}(\rho)(a, t) & \\
= & p_{0}(a-t) \pi_{1}(a, t, t, \rho)-r_{0}(a-t) \pi_{3}(a, t, t, \rho) \\
& +\int_{0}^{t} \pi_{1}^{1}(a, t, \sigma, \rho)\left(\widehat{\mu}_{1}(a-\sigma, t-\sigma, P(t-\sigma)) F_{2}(\rho)(a-\sigma, t-\sigma)\right. \\
& \left.+\widehat{\mu}_{2}(a-\sigma, t-\sigma, P(t-\sigma)) F_{3}(\rho)(a-\sigma, t-\sigma)\right) d \sigma \\
& -\int_{0}^{t} \gamma(a-\sigma) \pi_{3}(a, t, \sigma, \rho)\left(\rho_{1}(a-\sigma, t-\sigma)-F_{2}(\rho)(a-\sigma, t-\sigma)\right) d \sigma \\
= & : p_{0}(a-t) \pi_{1}(a, t, t, \rho)-r_{0}(a-t) \pi_{3}(a, t, t, \rho)+A_{1}+C_{1}+C_{2} .
\end{aligned}
$$

By the mean value theorem, there exists $t_{3}, t_{4} \in(0, t)$, such that

$$
\begin{aligned}
A_{1} & =\int_{0}^{t} \pi_{1}^{1}(a, t, \sigma, \rho) \widehat{\mu}_{1}(a-\sigma, t-\sigma, P(t-\sigma)) F_{2}(\rho)(a-\sigma, t-\sigma) d \sigma \\
& =\int_{0}^{t} \pi_{1}^{1}(a, t, \sigma, \rho) \widehat{\mu}_{1}(a-\sigma, t-\sigma, P(t-\sigma)) s_{0}(a-t) \pi_{2}(a-\sigma, t-\sigma, t-\sigma, \rho) d \sigma \\
& =s_{0}(a-t) \pi_{2}(a, t, t, \rho)\left(1-\Gamma_{1}(0, t)\right) \Psi_{1}^{-1}\left(0, t_{3}\right) \Psi_{2}^{-1}\left(0, t_{4}\right) .
\end{aligned}
$$


By the mean value theorem, we have the following:

$$
\begin{aligned}
C_{1}= & r_{0}(a-t) \int_{0}^{t} \pi_{1}^{1}(a, t, \sigma, \rho) \widehat{\mu}_{2}(a-\sigma, t-\sigma, P(t-\sigma)) \pi_{3}(a-\sigma, t-\sigma, t-\sigma, \rho) d \sigma \\
= & r_{0}(a-t) \pi_{3}(a, t, t, \rho)\left(1-\Gamma_{2}(0, t)\right), \\
C_{2}= & \int_{0}^{t} \pi_{1}^{1}(a, t, \sigma, \rho) \widehat{\mu}_{2}(a-\sigma, t-\sigma, P(t-\sigma)) \\
& \times \int_{\sigma}^{t} \gamma(a-s) \pi_{3}(a-\sigma, t-\sigma, s-\sigma, \rho)\left(\rho_{1}(a-s, t-s)-F_{2}(\rho)(a-s, t-s)\right) d s d \sigma \\
= & \int_{0}^{t} \gamma(a-s)\left(\rho_{1}(a-s, t-s)-F_{2}(\rho)(a-s, t-s)\right) \\
& \times \int_{0}^{s} \pi_{1}^{1}(a, t, \sigma, \rho) \widehat{\mu}_{2}(a-\sigma, t-\sigma, P(t-\sigma)) \pi_{3}(a-\sigma, t-\sigma, s-\sigma, \rho) d \sigma d s \\
= & \int_{0}^{t} \gamma(a-s)\left(\rho_{1}(a-s, t-s)-F_{2}(\rho)(a-s, t-s)\right) \pi_{3}(a, t, s, \rho) \int_{0}^{s}-\frac{d}{d \sigma} \Gamma_{2}(0, \sigma) d \sigma d s \\
= & \int_{0}^{t} \gamma(a-s)\left(\rho_{1}(a-s, t-s)-F_{2}(\rho)(a-s, t-s)\right) \pi_{3}(a, t, s, \rho)\left(1-\Gamma_{2}(0, s)\right) .
\end{aligned}
$$

Thus,

$$
\begin{aligned}
F_{1}(\rho)(a, t)-F_{3}(\rho)(a, t) & \\
= & p_{0}(a-t) \pi_{1}^{1}(a, t, t, \rho)-r_{0}(a-t) \pi_{3}(a, t, t, \rho) \\
& +\int_{0}^{t} \pi_{1}^{1}(a, t, \sigma, \rho) \widehat{\mu}_{1}(a-\sigma, t-\sigma, P(t-\sigma)) F_{2}(\rho)(a-\sigma, t-\sigma) d \sigma \\
& +r_{0}(a-t) \pi_{3}(a, t, t, \rho)\left(1-\Gamma_{2}(0, t)\right) \\
& \quad-\int_{0}^{t} \gamma(a-s)\left(\rho_{1}(a-s, t-s)-F_{2}(\rho)(a-s, t-s)\right) \pi_{3}(a, t, s, \rho)\left(1-\Gamma_{2}(0, s)\right) d s \\
\geq & \left(p_{0}(a-t)-r_{0}(a-t)\right) \pi_{1}^{1}(a, t, t, \rho) \\
& +\int_{0}^{t} \pi_{1}^{1}(a, t, \sigma, \rho) \widehat{\mu}_{1}(a-\sigma, t-\sigma, P(t-\sigma)) F_{2}(\rho)(a-\sigma, t-\sigma) d \sigma \geq 0 .
\end{aligned}
$$

So that $F_{1}(\rho)(a, t) \geq F_{2}(\rho)(a, t) \geq 0, F_{1}(\rho)(a, t) \geq F_{3}(\rho)(a, t) \geq 0$, a.e. $a \in(t,+\infty)$, and we can conclude that for each $\rho \in V, F(\rho) \in V$.

Theorem 3.4. Suppose $\left(H_{1}\right)-\left(H_{5}\right)$, for each $T>0$ and for each $\rho_{0}=\left(p_{0}, s_{0}, r_{0}\right) \in\left(L_{1}\left(R_{+}\right)\right)^{3}$, with $p_{0} \geq s_{0}, p_{0} \geq r_{0}$, there exists a unique $\rho=(p, s, r) \in V$ satisfying (3.2), (3.4), and (3.5), or (3.3), (3.4), and (3.5). And so, $\rho$ is the unique solution to problem (2.10). 
Proof. In order to prove the theorem, it remains to be shown that $F$ (defined by (3.11) and (3.18)) has a unique point fixed in $V$.

Let $C_{r, w}$ be defined by (3.7); then for $w$ being large enough $F$ maps $C_{r, w}$ into $C_{r, w}$. Indeed, by estimate of $|\rho(\cdot, \cdot)|_{1}$, we get, for almost all $t \in 1$

$$
\begin{aligned}
& |F(\rho)(\cdot, t)|_{1}=\left\|F_{1}(\rho)(\cdot, t)\right\|_{L^{1}}+\left\|F_{2}(\rho)(\cdot, t)\right\|_{L^{1}}+\left\|F_{3}(\rho)(\cdot, t)\right\|_{L^{1}} \\
& =\int_{t}^{+\infty}\left(p_{0}(a-t) \pi_{1}(a, t, t, \rho)+s_{0}(a-t) \pi_{2}(a, t, t, \rho)+r_{0}(a-t) \pi_{3}(a, t, t, \rho)\right) d a \\
& +\int_{0}^{t}\left(B_{p}(t-a) \pi_{1}(a, t, a, \rho)+B_{p}(t-a) \pi_{2}(a, t, a, \rho)\right) d a \\
& +\int_{0}^{t} \int_{0}^{a} \pi_{1}(a, t, \sigma, \rho)\left[\widehat{\mu}_{1}(a-\sigma, t-\sigma) F_{2}(\rho)(a-\sigma, t-\sigma)\right. \\
& \left.+\widehat{\mu}_{2}(a-\sigma, t-\sigma) F_{3}(\rho)(a-\sigma, t-\sigma)\right] d \sigma d a \\
& +\int_{t}^{+\infty} \int_{0}^{t} \pi_{1}(a, t, \sigma, \rho)\left[\widehat{\mu}_{1}(a-\sigma, t-\sigma) F_{2}(\rho)(a-\sigma, t-\sigma)\right. \\
& \left.+\widehat{\mu}_{2}(a-\sigma, t-\sigma) F_{3}(\rho)(a-\sigma, t-\sigma)\right] d \sigma d a \\
& +\int_{0}^{t} \int_{0}^{a} \gamma(a-\sigma) \pi_{3}(a, t, \sigma, \rho)\left[\rho_{1}(a-\sigma, t-\sigma)-F_{2}(\rho)(a-\sigma, t-\sigma)\right] d \sigma d a \\
& +\int_{t}^{+\infty} \int_{0}^{a} \gamma(a-\sigma) \pi_{3}(a, t, \sigma, \rho)\left[\rho_{1}(a-\sigma, t-\sigma)-F_{2}(\rho)(a-\sigma, t-\sigma)\right] d \sigma d a \\
& \leq \int_{t}^{+\infty}\left(\left|p_{0}(a-t)\right|+\left|s_{0}(a-t)\right|+\left|r_{0}(a-t)\right|\right) d a+2 \int_{0}^{t} B_{p}(t-a) d a \\
& +\int_{0}^{t} \int_{0}^{+\infty} \pi_{1}(a+t-\sigma, t, t-\sigma, \rho)\left[\widehat{\mu}_{1}(a, \sigma) F_{2}(\rho)(a, \sigma)+\widehat{\mu}_{2}(a, \sigma) F_{3}(\rho)(a, \sigma)\right] d a d \sigma \\
& +\int_{0}^{t} \int_{0}^{+\infty} \gamma(a-\sigma) \pi_{3}(a+t-\sigma, t, t-\sigma, \rho)\left[\rho_{1}(a, \sigma)-F_{2}(\rho)(a, \sigma)\right] d a d \sigma \\
& \leq\left|\rho_{0}(\cdot, t)\right|_{1}+\bar{\mu}_{1} \int_{0}^{t}\left\|F_{2}(\rho)(\cdot, u)\right\|_{L^{1}} d u+\bar{\mu}_{2} \int_{0}^{t}\left\|F_{3}(\rho)(\cdot, u)\right\|_{L^{1}} d u+\gamma_{\infty} \int_{0}^{t}\left\|\rho_{1}(\cdot, u)\right\| d u \\
& +\gamma_{\infty} \int_{0}^{t}\left\|F_{2}(\rho)(\cdot, u)\right\|_{L^{1}} d u \\
& \leq\left|\rho_{0}(\cdot, t)\right|_{1}+\left(\bar{\mu}_{1}+2 \gamma_{\infty}+\bar{\mu}_{2}+2 C_{4}(T)\right) \int_{0}^{t} \log (|P|+e)\|\rho(\cdot, u)\|_{L^{1}} d u .
\end{aligned}
$$

And from Gronwall's inequality, it follows that

$$
|F(\rho)(\cdot, t)|_{1} \leq \exp \left(r e^{w t}\right), \quad \text { a.e. } t \in I,
$$


for $w>0$ depending on $T, \bar{\mu}_{1}, \bar{\mu}_{2}$, and $\gamma_{\infty}$. Hence, we have proved that $F$ maps $C_{r, w}$ into $C_{r, w}$.

Let us assume that $w$ is fixed such that $F(\rho)$ remains in $C_{r, w}$ for $\rho$ in $C_{r, w}$. Clearly, $C_{r, w}$ is closed in $V$ and to prove that $F$ has a unique fixed point in $C_{r, w}$, it suffices to prove that $F$ is a strict contraction, for instance for the norm defined in definition of $|\rho|_{V}$ with $k$ suitable. For convenience in the following we denote $M$ a certain (which may change) but which is independent of $a, t$ and $P(t)$. For $\rho:=(p, s, r), \rho^{\prime}:=\left(p^{\prime}, s^{\prime}, r^{\prime}\right) \in C_{r, w}$, let us estimate $\left|F(\rho)-F\left(\rho^{\prime}\right)\right|_{V}$.

First, for almost all $t \in I$,

$$
\begin{aligned}
\left|F(\rho)-F\left(\rho^{\prime}\right)\right|_{1}= & \int_{0}^{+\infty}\left|F_{1}(\rho)(a, t)-F_{1}\left(\rho^{\prime}\right)(a, t)\right| d a+\int_{0}^{+\infty}\left|F_{2}(\rho)(a, t)-F_{2}\left(\rho^{\prime}\right)(a, t)\right| d a \\
& +\int_{0}^{+\infty}\left|F_{3}(\rho)(a, t)-F_{3}\left(\rho^{\prime}\right)(a, t)\right| d a \\
= & f_{1}(t)+f_{2}(t)+f_{3}(t) .
\end{aligned}
$$

Now, substituting the expression of $F_{1}$ into $f_{1}$, we get

$$
\begin{aligned}
f_{1}(t) \leq & \int_{0}^{t}\left|B_{p}(t-a) \pi_{1}(a, t, a, \rho)-B_{p^{\prime}}(t-a) \pi_{1}\left(a, t, a, \rho^{\prime}\right)\right| d a \\
& +\int_{t}^{+\infty}\left|p_{0}(a-t)\right|\left|\pi_{1}(a, t, t, \rho)-\pi_{1}\left(a, t, t, \rho^{\prime}\right)\right| d a \\
& +\int_{0}^{t} \int_{0}^{\infty} \mid \pi_{1}(a+t-\sigma, t, t-\sigma, \rho) \widehat{\mu}_{1}(a, \sigma, P(\sigma)) F_{2}(\rho)(a, \sigma) \\
& \quad-\pi_{1}\left(a+t-\sigma, t, t-\sigma, \rho^{\prime}\right) \widehat{\mu}_{1}\left(a, \sigma, P^{\prime}(\sigma)\right) F_{2}\left(\rho^{\prime}\right)(a, \sigma) \mid d a d \sigma \\
& +\int_{0}^{t} \int_{0}^{\infty} \mid \pi_{1}(a+t-\sigma, t, t-\sigma, \rho) \widehat{\mu}_{2}(a, \sigma, P(\sigma)) F_{3}(\rho)(a, \sigma) \\
& -\pi_{1}\left(a+t-\sigma, t, t-\sigma, \rho^{\prime}\right) \widehat{\mu}_{2}\left(a, \sigma, P^{\prime}(\sigma)\right) F_{3}\left(\rho^{\prime}\right)(a, \sigma) \mid d a d \sigma \\
= & f_{1}^{1}(t)+f_{1}^{2}(t)+f_{1}^{3}(t)+f_{1}^{4}(t),
\end{aligned}
$$

where $P(t):=\int_{0}^{+\infty} p(a, t) d a$ and $P^{\prime}(t):=\int_{0}^{+\infty} p^{\prime}(a, t) d a$. Hence

$$
\begin{aligned}
f_{1}^{2}(t) & =\int_{t}^{\infty}\left|p_{0}(a-t)\right|\left|\pi_{1}(a, t, a, \rho)-\pi_{1}\left(a, t, a, \rho^{\prime}\right)\right| d a \\
& \leq \frac{C(T)}{k} e^{k t}\left|\rho-\rho^{\prime}\right| \int_{t}^{+\infty}\left|p_{0}(a-t)\right| d a \\
& \leq \frac{C(T)}{k}\left|\rho-\rho^{\prime}\right|_{V}\left\|\rho_{0}\right\|_{L^{1}} e^{k t}
\end{aligned}
$$


Estimate of $f_{1}^{1}$. By (3.10), we have

$$
\begin{aligned}
f_{1}^{1}(t) \leq & \int_{0}^{t}\left|B_{p}(t-a)\right|\left|\pi_{1}(a, t, a, \rho)-\pi_{1}\left(a, t, a, \rho^{\prime}\right)\right| d a \\
& +\int_{0}^{t}\left|B_{p}(t-a)-B_{p^{\prime}}(t-a)\right|\left|\pi_{1}\left(a, t, a, \rho^{\prime}\right)\right| d a \\
:= & f_{1}^{11}+f_{1}^{12} .
\end{aligned}
$$

Let us now estimate $f_{1}^{11}$. By (3.9) and (3.10), we get

$$
f_{1}^{11} \leq \frac{M C(T)}{k}\left|\rho-\rho^{\prime}\right|_{V} e^{k t}
$$

Let us estimate $f_{1}^{12}(t)$. Thanks to (2.7), (2.8), and (3.8), we have

$$
\begin{aligned}
f_{1}^{12}(t) \leq & \int_{0}^{t}\left|B_{p}(t-a)-B_{p^{\prime}}(t-a)\right| d a=\int_{0}^{t}\left|B_{p}(u)-B_{p^{\prime}}(u)\right| d u \\
\leq & \int_{0}^{t} \int_{0}^{+\infty}|\beta(a, u, P(u))|\left|p(a, u)-p^{\prime}(a, u)\right| d a d u \\
& +\int_{0}^{t} \int_{0}^{+\infty}\left|\beta(a, u, P(u))-\beta\left(a, u, P^{\prime}(u)\right)\right|\left|p^{\prime}(a, u)\right| d a d u \\
\leq & \int_{0}^{t} C_{4}(T) \log (|P|+e)\left\|p(\cdot, u)-p^{\prime}(\cdot, u)\right\|_{L^{1}} d u+\int_{0}^{t} C_{3}(T) M\left\|p(\cdot, u)-p^{\prime}(\cdot, u)\right\|_{L^{1}} d u \\
\leq & \left(C_{4}(T) \log (|P|+e)+C_{3}(T) M\right)\left\|p(\cdot, u)-p^{\prime}(\cdot, u)\right\|_{L^{1}} d u \\
\leq & M \int_{0}^{t}\left|\rho-\rho^{\prime}\right|_{1} d u \leq \frac{M}{k}\left|\rho-\rho^{\prime}\right|_{V} e^{k t} .
\end{aligned}
$$

Second, let us estimate $f_{2}$. Substituting the expression for $F_{2}$ into $f_{2}$ and applying (3.11), we obtain

$$
\begin{aligned}
f_{2}(t) \leq & \int_{t}^{+\infty}\left|s_{0}(a-t)\right|\left|\pi_{2}(a, t, a, \rho)-\pi_{2}\left(a, t, a, \rho^{\prime}\right)\right| d a \\
& +\int_{0}^{t}\left|B_{p}(t-a)\right|\left|\pi_{2}(a, t, a, \rho)-\pi_{2}\left(a, t, a, \rho^{\prime}\right)\right| d a \\
\leq & \frac{C\left(T, K_{\infty}\right)\left\|s_{0}\right\|_{L^{1}}}{k}\left|\rho-\rho^{\prime}\right|_{V} e^{k t}+\frac{M C\left(T, K_{\infty}\right)}{k}\left|\rho-\rho^{\prime}\right|_{V} e^{k t}+\frac{M}{k}\left|\rho-\rho^{\prime}\right|_{V} e^{k t} \\
\leq & \frac{M}{k}\left|\rho-\rho^{\prime}\right|_{V} e^{k t} .
\end{aligned}
$$


Estimate of $f_{1}^{3}$. By (2.6) and (3.8), $\left|\widehat{\mu}_{i}(a, t, P(t))\right| \leq \bar{\mu}_{i}, i=1,2$, then

$$
\begin{aligned}
f_{1}^{3}(t) \leq & \bar{\mu}_{1} \int_{0}^{t} \int_{0}^{+\infty}\left|\pi_{1}(a+t-\sigma, t, t-\sigma, \rho)\right|\left|F_{2}(\rho)(a, \sigma)-F_{2}\left(\rho^{\prime}\right)(a, \sigma)\right| d a d \sigma \\
& +\bar{\mu}_{1} \int_{0}^{t} \int_{0}^{+\infty}\left|\pi_{1}(a+t-\sigma, t, t-\sigma, \rho)-\pi_{1}\left(a+t-\sigma, t, t-\sigma, \rho^{\prime}\right)\right|\left|F_{2}\left(\rho^{\prime}\right)(a, \sigma)\right| d a d \sigma \\
& +\int_{0}^{t} \int_{0}^{+\infty}\left|\pi_{1}(a+t-\sigma, t, t-\sigma, \rho)\right|\left|F_{2}\left(\rho^{\prime}\right)(a, \sigma)\right|\left|\widehat{\mu}_{1}(a, \sigma, P(\sigma))-\widehat{\mu}_{1}\left(a, \sigma, P^{\prime}(\sigma)\right)\right| d a d \sigma .
\end{aligned}
$$

Since $F_{2}(\rho) \in C_{r, w}$, we have $\left\|F_{2}(\rho)\right\|_{L^{1}} \leq M$, and then

$$
\begin{aligned}
f_{1}^{3}(t) & \leq \frac{\bar{\mu}_{1}}{k}\left|\rho-\rho^{\prime}\right|_{V} e^{k t}+\frac{M C_{1}(T)}{k^{2}}\left|\rho-\rho^{\prime}\right|_{V} e^{k t}+\frac{M C_{1}(T)}{k}\left|\rho-\rho^{\prime}\right|_{V} e^{k t} \\
& =\left(\frac{M}{k^{2}}+\frac{M}{k}\right)\left|\rho-\rho^{\prime}\right|_{V} e^{k t} .
\end{aligned}
$$

Finally, let us estimate of $f_{3}$.

$$
\begin{aligned}
f_{3}(t) \leq & \int_{t}^{+\infty}\left|r_{0}(a-t)\right|\left|\pi_{3}(a, t, a, \rho)-\pi_{3}\left(a, t, a, \rho^{\prime}\right)\right| d a \\
& +\int_{0}^{t} \int_{0}^{+\infty} r(a-\sigma) \mid \pi_{3}(a+t-\sigma, t, t-\sigma, \rho)\left(F_{1}(\rho)(a, \sigma)-F_{2}(\rho)(a, \sigma)\right) \\
\leq & \frac{C_{3}(T)\left\|r_{0}\right\|_{L^{1}}\left|\rho-\rho^{\prime}\right|_{V} e^{k t}+r_{\infty}}{k} \\
& \times \int_{0}^{t} \int_{0}^{+\infty}\left|F_{1}(\rho)(a, \sigma)\right|\left|\pi_{3}(a+t-\sigma, t, t-\sigma, \rho)-\pi_{3}\left(a+t-\sigma, t, t-\sigma, \rho^{\prime}\right)\right| d a d \sigma \\
& +\gamma_{\infty} \int_{0}^{t} \int_{0}^{+\infty}\left|\pi_{3}\left(a+t-\sigma, t, t-\sigma, \rho^{\prime}\right)\right|\left|F_{1}(\rho)(a, \sigma)-F_{1}\left(\rho^{\prime}\right)(a, \sigma)\right| d a d \sigma \\
& +r_{\infty} \int_{0}^{t} \int_{0}^{+\infty}\left|F_{2}(\rho)(a, \sigma)\right|\left|\pi_{3}(a+t-\sigma, t, t-\sigma, \rho)-\pi_{3}\left(a+t-\sigma, t, t-\sigma, \rho^{\prime}\right)\right| d a d \sigma \\
& +r_{\infty} \int_{0}^{t} \int_{0}^{+\infty}\left|\pi_{3}\left(a+t-\sigma, t, t-\sigma, \rho^{\prime}\right)\right| \\
& \times\left|F_{2}(\rho)(a, \sigma)-F_{2}\left(\rho^{\prime}\right)(a, \sigma)\right| d a d \sigma \\
\leq & \left(\frac{C_{3}(T)\left\|r_{0}\right\|_{L^{1}}}{k}+\frac{\gamma_{\infty} C_{3}(T) M}{k^{2}}+\frac{r_{\infty}}{k}+\frac{r_{\infty} M}{k^{2}}+\frac{r_{\infty} M}{k^{2}}\right)\left|\rho-\rho^{\prime}\right|_{V} e^{k t} \\
= & \left(\frac{M}{k^{2}}+\frac{M}{k}\right)\left|\rho-\rho^{\prime}\right|_{V} e^{k t} .
\end{aligned}
$$




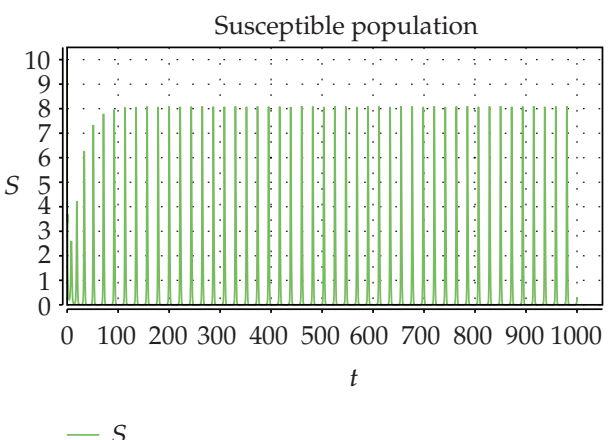

(a)

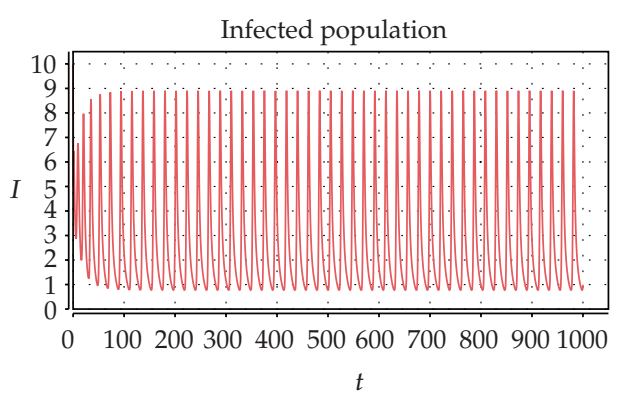

(b)

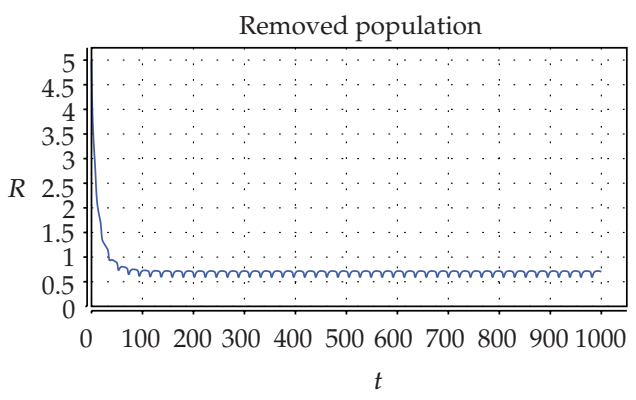

$R$

(c)

Figure 1: The temporal solution found by numerical integration of problem with initial values $s_{0}(a)=10$, $i_{0}(a)=10, r_{0}(a)=5$. They show that system (2.2) has a unique positive periodic solution.

Therefore, joining all above estimates, we see that for almost all $t \in 1$, there exist $M>0$ and $\widehat{M}>0$ depending only on $\rho_{0}, T, \gamma_{\infty}, \bar{\mu}_{i}, i=1,2$, and $K_{\infty}$, such that

$$
\left|F(\rho)(\cdot, t)-F\left(\rho^{\prime}\right)(\cdot, t)\right|_{1} \leq\left(\frac{M}{k}+\frac{\widehat{M}}{k^{2}}\right)\left|\rho-\rho^{\prime}\right|_{V} e^{k t} .
$$

Dividing both sides of this inequality by $e^{k t}$, we obtain

$$
\left|F(\rho)(\cdot, t)-F\left(\rho^{\prime}\right)(\cdot, t)\right|_{V} \leq\left(\frac{M}{k}+\frac{\widehat{M}}{k^{2}}\right)\left|\rho-\rho^{\prime}\right|_{V} .
$$

And thus for $k$ great enough $F$ is a strict contraction with a unique fixed point in $C_{r, w}$, and so in $V$. This concludes the proof.

\section{Discussion}

In this paper, existence of positive period solution of a non-autonomous SIR epidemic model with age structure is studied. We obtained existence and uniqueness of this model using integral differential equation and a fixed theorem. The model is different from the classical 
age structure epidemic model and non-autonomous epidemic model. The initial condition is nonlocal and dependent on total population. In addition, incidence law is not Lipschitzianity. The classical methods are not valid. We construct a new norm and prove the existence of our model under definition of the new norm. We can illustrate this through two simulates examples. We set

$$
\begin{gathered}
\beta(a, t, P(t))=\left\{\begin{array}{ll}
0, & 0<a<1, \\
4\left(1-\frac{P(t)}{13400}\right), & a \geq 1 .
\end{array} \quad \lambda(a, t, i)= \begin{cases}0, & 0<a<1, \\
\frac{4.86}{P(t)}, & a \geq 1 .\end{cases} \right. \\
\mu_{1}(a, t, P(t))=0.02 P^{2}(t), \quad \mu_{2}(a, t, P(t))=0.05 P(t), \\
\mu_{3}(a, t, P(t))=0.01 P(t), \quad \gamma(a)= \begin{cases}0, & 0<a<1, \\
0.01, & a \geq 1 .\end{cases}
\end{gathered}
$$

System (2.2) with above coefficients has a unique positive periodic solution. We can see it from Figure 1.

In the future, there are some problems that will be solved. The existence of steady state and stability of the steady state are still discussed. If birth rate is impulsive, what results will occur. The simulation of the age structure still to be resolved. Furthermore, what effect will occurs, if we introduce the delay in our model.

\section{Acknowledgments}

This work is Supported by the National Sciences Foundation of China (10971178), the Sciences Foundation of Shanxi (20090110053), and the Sciences Exploited Foundation of Shanxi (20081045).

\section{References}

[1] A. J. Lotka, Elements of Mathematical Biology, Dover, New York, NY, USA, 1956.

[2] H. Von Foerster, "Some remarks on changing populations," in The Kinetics of Cellular Proliferation, pp. 382-407, Grune \& Stratton, New York, NY, USA, 1959.

[3] M. E. Gurtin and R. C. MacCamy, "Non-linear age-dependent population dynamics," Archive for Rational Mechanics and Analysis, vol. 54, pp. 281-300, 1974.

[4] S. N. Busenberg, M. Iannelli, and H. R. Thieme, "Global behavior of an age-structured epidemic model," SIAM Journal on Mathematical Analysis, vol. 22, no. 4, pp. 1065-1080, 1991.

[5] M. Iannelli, F. A. Milner, and A. Pugliese, "Analytical and numerical results for the age-structured S-IS epidemic model with mixed inter-intracohort transmission," SIAM Journal on Mathematical Analysis, vol. 23, no. 3, pp. 662-688, 1992.

[6] M. El-Doma, "Analysis for an SIR age-structured epidemic model with vertical transmission and vaccination," International Journal of Ecology and Development, vol. 3, pp. 1-38, 2005.

[7] G. F. Webb, Theory of Nonlinear Age-Dependent Population Dynamics, vol. 89 of Monographs and Textbooks in Pure and Applied Mathematics, Marcel Dekker, New York, NY, USA, 1985.

[8] H. R. Thieme, "Uniform weak implies uniform strong persistence for non-autonomous semiflows," Proceedings of the American Mathematical Society, vol. 127, no. 8, pp. 2395-2403, 1999.

[9] W. Wang and X.-Q. Zhao, "Threshold dynamics for compartmental epidemic models in periodic environments," Journal of Dynamics and Differential Equations, vol. 20, no. 3, pp. 699-717, 2008. 
[10] T. Zhang and Z. Teng, "Permanence and extinction for a nonautonomous SIRS epidemic model with time delay," Applied Mathematical Modelling, vol. 33, no. 2, pp. 1058-1071, 2009.

[11] Z. Teng, Y. Liu, and L. Zhang, "Persistence and extinction of disease in non-autonomous SIRS epidemic models with disease-induced mortality," Nonlinear Analysis: Theory, Methods E Applications, vol. 69, no. 8, pp. 2599-2614, 2008.

[12] M. Delgado, M. Molina-Becerra, and A. Suárez, "Analysis of an age-structured predator-prey model with disease in the prey," Nonlinear Analysis: Real World Applications, vol. 7, no. 4, pp. 853-871, 2006. 